\title{
El Servicio Profesional Docente y las licenciaturas en ciencias de la educación. Elementos para el análisis
}

Graciela Cordero-Arroyo y Ana-María Salmerón-Castro

\section{RESUMEN}

Entre las modificaciones legislativas que han acompañado a la nueva reforma educativa mexicana, el artículo 24 de la Ley General del Servicio Profesional Docente ha abierto la posibilidad de que nuevas profesiones con perfiles pedagógicos o afines se incorporen a las tareas magisteriales en los niveles de preescolar y primaria. La nueva disposición legislativa da por sentada una suerte de homologación de los perfiles profesionales de maestros normalistas y egresados de licenciaturas en educación o pedagogía. El texto que presentamos intenta, por un lado, dar cuenta de algunas de las razones lógicas y empíricas que resisten la posibilidad de afirmar tal homologación; por otro lado, avanza en la previsión de algunas de las consecuencias esperables de la aplicación de esta disposición jurídica. El análisis se ilustra con la revisión de los resultados de la convocatoria de ingreso al Servicio Profesional Docente de los egresados de la licenciatura en ciencias de la educación de una universidad pública estatal.

Palabras clave: reforma educativa, educación básica, formación inicial de profesores, formación de pedagogos, México.

Graciela Cordero-Arroyo

Mexicana. Doctora en Filosofía y Ciencias de la Educación por la Universidad de Barcelona. Investigadora titular del Instituto de Investigación y Desarrollo Educativo, Universidad Autónoma de Baja California. Temas de investigación: formación de profesores y políticas docentes.

Ana-María Salmerón-Castro

anasalme@unam.mx Mexicana. Doctora en Pedagogía por la Universidad Nacional Autónoma de México. Profesora de carrera de la Facultad de Filosofía y Letras, Universidad Nacional Autónoma de México. Temas de investigación: educación moral y política; desigualdad social y justicia educativa. 


\title{
O serviço profissional docente e as licenciaturas em ciências da educação. Elementos para a análise
}

\section{RESUMO}

Entre as modificações legislativas que têm acompanhado a nova reforma educativa mexicana, o artigo 24 da Lei Geral do Serviço Profissional Docente tem aberto a possibilidade de que novas profissões com perfis pedagógicos ou afins se incorporem as tarefas docentes nos níveis de pré-escolar e ensino fundamental. A nova disposição legislativa assume uma homologação dos perfis profissionais de professores formados em magistério e licenciaturas em educação ou pedagogia. O texto que apresentamos tenta, por um lado, entender algumas das razões lógicas e empíricas que resistem a possibilidade de afirmar tal homologação; por outro lado, avança na previsão de algumas das consequências esperáveis da aplicação desta disposição jurídica. A análise se ilustra com a revisão dos resultados da convocatória de ingresso ao Serviço Profissional Docente dos recém-formados da licenciatura em ciências da educação de uma universidade pública estatal.

Palavras chave: reforma educativa, educação básica, formação inicial de professores, formação de pedagogos, México.

\section{The Professional teaching service and the degrees in educational sciences. Elements for an analysis}

\begin{abstract}
Amongst the legislative changes that have accompanied the new Mexican educational reform, the article 24 of the General Law for Professional Teaching Service (Ley General del Servicio Profesional Docente) has given rise to the possibility that new professions with pedagogical profile get involved in teaching tasks in preschool and elementary education. The new legislative decree takes for granted that the professional profiles of teachers that were trained in teacher-training colleges and students who graduated from degrees in education or pedagogy can be seen as equivalent. This article seeks, on one hand, to give an account of the logical and empirical reasons that make impossible that kind of standardization; on the other hand, it foresees some of the expected consequences that would cause the implementation of this legal provision. The analysis is illustrated by a review of the results at the call for applying at the Professional Teaching Service (Servicio Profesional Docente) of the students who graduated from Educational Sciences degree in a university situated in a federate state of the Mexican Republic.
\end{abstract}

Key words: educational reform, basic education, initial education for teachers, training of educators, Mexico.

Recepción: 20/05/16. Aprobación: 10/08/16. 


\section{Introducción}

La Reforma del sistema educativo mexicano fue anunciada el primero de diciembre de 2012, el primer día de gobierno del actual presidente de la República. Desde ese día y hasta hoy, la prensa, otros medios de comunicación y la academia han dado cuenta de análisis y cuestionamientos de los más variados tintes sobre la regulación de la carrera docente.

El debate sobre la Reforma Educativa se ha centrado, principalmente, en el marco de la evaluación docente, la cara más visible del proyecto de cambio educativo propuesto al inicio del sexenio. Existen, hay que considerar, otras implicaciones de la reforma que aún demandan atención y reflexión. La agenda de la reforma y las posibilidades de sus consecuencias tienen pendiente un amplio abanico de temas de estudio y debate.

El tema que proponemos en este trabajo es la posibilidad que la Ley General del Servicio Profesional Docente (LGSPD) abre para que los egresados de las instituciones de educación superior (IES) puedan ingresar al Servicio Profesional Docente (SPD) como maestros de educación preescolar y primaria. Esta opción, aprobada por la LGSPD en septiembre de 2013, fue recientemente incluida en la agenda de discusión sobre la Reforma Educativa a partir del pronunciamiento que hizo el secretario de Educación, Aurelio Nuño, en la Cámara de Senadores el pasado 22 de marzo de 2016.

La LGSPD establece que un egresado universitario puede ser docente de educación básica siempre y cuando acredite el examen de ingreso y define, como única condición para ingresar al servicio, tener formación docente pedagógica o en áreas afines. Esto nos lleva a asumir que la Ley hace referencia específicamente a los licenciados en pedagogía, en ciencias de la educación y en educación que egresan de las IES públicas y privadas del país.

Este artículo tiene como objetivo analizar la manera en que el enfoque meritocrático de la carrera docente de profesores de educación preescolar y primaria y su regulación en el marco de la LGSPD afecta, o puede afectar, las comprensiones respecto del papel de las licenciaturas en ciencias de la educación y pedagogía que ofrecen las IES y el significado de la propia tarea magisterial. El análisis se ilustra con la revisión de los resultados de la convocatoria de ingreso al SPD de los egresados de la licenciatura en ciencias de la educación de una universidad pública estatal.

Hemos organizado el artículo en tres apartados generales. El primero atiende al enfoque meritocrático de la carrera docente mexicana y la manera en que la Ley posibilita que los egresados de estas licenciaturas puedan ingresar al servicio como maestros de educación preescolar y primaria. El segundo apartado enfoca el análisis de las carreras consideradas afines al normalismo. Aquí presentamos el examen de los resultados de los aspirantes de la licenciatura en Ciencias de la Educación de la Universidad Autónoma de Baja California (UABC) al SPD en los ciclos 2014-2015 y 2015-2016. El tercer apartado plantea la discusión respecto de la pertinencia de la disposición legal que asume como equivalentes la formación magisterial y la de los profesionistas en pedagogía o en ciencias de la educación y da cuenta de las potenciales consecuencias a corto, mediano y largo plazos.

\section{Enfoque meritocrático de la carrera docente mexicana}

Declarativamente, las autoridades han asignado a la reforma la voluntad de búsqueda de optimizar la "calidad de la educación". A las particularidades emblemáticas de la educación en México (la gratuidad, la laicidad, la obligatoriedad y el carácter democrático y nacional), se ha añadido la de la "calidad". Así se inscribe en la reciente modificación constitucional que ha abierto la puerta a la propuesta de reforma. El tercer artículo de nuestra Carta Magna, hoy incluye estos términos: "El Estado garantizará la calidad en 
la educación obligatoria de manera que los materiales y métodos educativos, la organización escolar, la infraestructura educativa y la idoneidad de los docentes y los directivos garanticen el máximo logro de aprendizaje de los educandos (adicionado mediante decreto publicado en el Diario Oficial de la Federación el 26 de febrero de 2013)" (DOF, 2013a: 1).

El Instituto para la Evaluación de la Educación (INEE) concibe la importancia de dichos elementos en un registro que asocia la noción de calidad directamente con el derecho fundamental a la educación. Los maestros, sostiene, son los primeros y más directos responsables del cumplimiento cabal de ese derecho:

La Reforma constitucional de 2013 incorpora el concepto de calidad como una característica esencial de la educación, definida como un derecho humano de todos. La importancia de los docentes en el Sistema Educativo Nacional (SEN) radica en su capacidad para concretar dicho derecho. Representan el primer punto de contacto del sistema educativo con los niños y jóvenes, así como con sus familias, y son corresponsables de mejorar los aprendizajes de sus alumnos. Por ello, los docentes son un factor clave en las reformas recientes al SEN y están en el centro de atención de las políticas públicas (INEE, 2015a: 9).

De cara a tal asignación respecto de la responsabilidad magisterial, la reforma instrumentó - entre sus primeras acciones - el establecimiento de una regulación de la trayectoria de los maestros vía el llamado "Servicio Profesional Docente (SPD)". ${ }^{1}$
Cuatro procesos lo integran: ingreso, promoción, permanencia y reconocimiento.

La LGSPD insertó, en la trayectoria de los docentes, un único criterio de selección: la demostración de las capacidades del individuo estimadas en una acción denominada evaluación del desempeño, es decir la valoración de sus méritos para ingresar, promocionarse o permanecer en el sistema por medio de diversos tipos de exámenes, de ahí la conceptualización de la carrera docente como meritocrática (Cuenca, 2015).

No obstante, los criterios que ha de tener el SPD para reconocer las cualidades del aspirante en servicio se topan con dificultades dignas de consideración. ${ }^{2}$ De acuerdo con el INEE:

El SPD, en los términos en que ha sido propuesto tanto en la Constitución como en la LGSPD, hace por lo menos tres grandes aportes: la instalación del mérito como principio rector para el ingreso y el desarrollo de la carrera magisterial, el reconocimiento integral a los docentes como sujetos plenos de derecho, y la aplicación de la norma de transparencia en relación con los criterios de evaluación. Tales aportes implican que el reclutamiento se abra a todos los candidatos que reúnan los requisitos exigidos, lo cual se acompaña de procedimientos y mecanismos tanto para evitar la arbitrariedad, la politización y las prácticas de patronazgo o clientelismo, así como para garantizar la igualdad efectiva y la no discriminación, orientadas a la superación de las desventajas - de cualquier tipo- en los procesos de incorporación, ascenso, reconocimiento o permanencia en el servicio. De tal forma, los

\footnotetext{
${ }^{1}$ Antes de la reforma educativa, los programas que definían el tránsito por el servicio docente y sus respectivos requisitos eran: el Escalafón (1973) y el Programa Nacional de Carrera Magisterial (en sus versiones de 1993, 2001 y 2011). Uno y otro reconocían la antigüedad en el servicio y la suma de diplomas, constancias, certificados y títulos acumulados. No estaba ausente, además, la cercanía a la autoridad sindical como un recurso de ingreso o movilidad al interior de la estructura; obviamente, como parte de la trama de normas no escritas al respecto. La reforma eliminó estos programas y su nueva legislación los transformó.

${ }^{2}$ No es propósito de este artículo la atención a muchas de ellas; sólo nos ocupamos de la que orienta la preocupación relacionada con las condiciones que se juzgan necesarias para las mejores posibilidades de la práctica de la enseñanza y los requisitos formativos que éstas imponen.
} 
aspirantes y servidores que se incorporen al servicio mediante concurso ganarán legitimidad, en tanto que sus nombramientos serán otorgados con base en sus propias cualidades (INEE, 2015a: 12).

En atención al reconocimiento de que serían tales las ventajas del nuevo recorrido de las trayectorias magisteriales en educación básica, la LGSPD estableció el Marco General de Galidad de la Educación (MGCE) para la evaluación del desempeño docente para el ingreso, promoción y permanencia, y estableció los referentes a partir de los cuales se juzga el "buen" desempeño docente. El marco está integrado, así, por el conjunto de "perfiles, parámetros e indicadores que se establecen como referentes para los concursos de oposición y la evaluación obligatoria para el ingreso, la promoción, el reconocimiento y la permanencia en el servicio" (artículo 4, fracción XVII) (DOF, 2013b: 3). ${ }^{3}$

De los cuatro procesos establecidos en la LGSPD, en este artículo nos centraremos en el de ingreso al SPD. El artículo 21 de la Ley - en tanto derivación directa de la reforma del artículo $3^{\circ}$ Constitucional- establece que el ingreso al servicio se llevará a cabo mediante concursos de oposición públicos "que garanticen la idoneidad de los conocimientos y las capacidades necesarias" (DOF, 2013b: 6). Dicha idoneidad se determina por el cumplimiento de los perfiles, parámetros e indicadores (PPI) de ingreso para docentes y técnicos docentes de todos los niveles educativos (SEP-CNSPD, 2015).

Por su parte, el artículo 24 de la ley que reglamenta el SPD establece que para ingresar al servicio pueden concursar:
[...] todas las personas que cumplan con el perfil relacionado con el nivel, tipo, modalidad y materia educativa correspondiente; así como con los requisitos que establezca la convocatoria respectiva, en igualdad de condiciones, sin demérito de origen, residencia, lugar o formación profesional. En la Educación Básica dicho perfil corresponderá al académico con formación docente pedagógica o áreas afines que corresponda a los niveles educativos, privilegiando el perfil pedagógico docente de los candidatos; también se considerarán perfiles correspondientes a las disciplinas especializadas de la enseñanza (DOF, 2013b: 7).

Esto implica un cambio en la condición de ingreso de los maestros de los niveles preescolar y primaria, la cual fue, antes de la reforma, la de ser egresado de las escuelas normales. El actual encuadre meritocrático de la carrera docente elimina este requisito de la enseñanza en el nivel básico y abre la opción a otros profesionales de la educación. Esto se observa en la segunda parte del artículo 24 de la LGSPD, donde se declara que el perfil del profesorado de educación básica debe corresponder al "académico con formación docente pedagógica o áreas afines". Desde nuestra perspectiva, esta matización del artículo 24 abre la posibilidad de que egresados universitarios de las carreras de ciencias de la educación y pedagogía puedan concursar por ingresar al nivel de educación preescolar y primaria, y debería ser una suerte de candado para egresados universitarios de otras áreas disciplinarias que no están contemplados en el artículo 24 de la Ley. ${ }^{4}$

\footnotetext{
${ }^{3}$ A la fecha, se han publicado los perfiles, parámetros e indicadores (PPI) de ingreso, de promoción y de permanencia de los actores educativos para todos los niveles educativos y modalidades (por ejemplo, SEP, 2016). El planteo meritocrático puede reconocerse - con cierta claridad - tanto en la trama de los perfiles como en la propia legislación relativa al ingreso al SPD.

${ }^{4}$ El caso de la educación secundaria es distinto. Históricamente, en nuestro país, la formación normalista no ha sido un requisito de ingreso para la incorporación a la tarea docente de escuela secundaria. Los maestros de este nivel se han incorporado al servicio magisterial, bien desde una formación provista en las escuelas normales, en la normal superior, o bien, en las más diversas instituciones universitarias. Es decir, la tradición de la enseñanza secundaria se ha construido a manos tanto de profesores normalistas como de egresados de los estudios profesionales que se ofrecen en las universidades sobre diferentes disciplinas. La heterogeneidad del magisterio en la escuela secundaria es, tradicionalmente, muy acentuada en nuestro país (Sandoval, 2009).
} 
En resumen, el título de la licenciatura en educación preescolar o primaria deja de ser - como era- el requisito indispensable para ingresar a la carrera docente en estos niveles educativos. Para ingresar al servicio magisterial servirá tanto un título obtenido en las escuelas normales como el de cualquier otra licenciatura que otorgue "formación docente pedagógica" o que privilegie el "perfil pedagógico docente". De esta forma, el sistema educativo mexicano define vías adicionales para acceder a la profesión y da lugar a que profesionistas de otras áreas educativas se desempeñen en las tareas de enseñanza de estos dos niveles educativos vía el concurso de ingreso.

En una etapa preparatoria a la aplicación de esta disposición, por otra parte, se estableció un artículo de vigencia perentoria en la LGSPD. Se trata del artículo vigésimo primero transitorio que establece que el artículo 24 entrará en vigor a los dos años siguientes a su publicación en el Diario Oficial de la Federación (es decir, en septiembre de 2015). Mientras tanto, la Ley aclara que las convocatorias para concursos de oposición para el Ingreso a la Educación Básica serán sólo para los egresados de las normales, pero permite que se abran convocatorias públicas para el caso de que el número de egresados con resultados idóneos de las normales no cubran las vacantes requeridas por las necesidades del propio servicio (DOF, 2013b).

Así, es en el marco de esta novedosa condición de la carrera docente, donde pretendemos incluir la preocupación que nos convoca al escribir este artículo.

\section{Carreras afines al normalismo para ingresar al SPD en educación preescolar y primaria}

Entre los "perfiles pedagógicos docentes" alternos, la SEP y el INEE han considerado a los egresados de las carreras que, genéricamente, se estipulan como "afines al normalismo" o "afines a las ciencias de la educación".
De acuerdo con datos del Comité para la Evaluación de Programas de Pedagogía y Educación (CEPPE) (2015), existen en el país alrededor de 1200 programas de carreras afines a las ciencias de la educación. El INEE (2015a) señala que estos programas tienen alrededor de 92000 estudiantes matriculados y que las licenciaturas relacionadas con la formación docente se imparten en 695 planteles en el país (2015b). En este grupo de programas se encuentran dos tipos de licenciaturas: 1) aquéllas centradas en una didáctica específica, es decir orientadas a la formación de maestros en una disciplina particular, y 2) las licenciaturas generales relacionadas con el área de pedagogía y ciencias de la educación (INEE, 2015b).

En este artículo nos ocuparemos sólo de las licenciaturas generales en el campo educativo. Se trata de las licenciaturas en educación, en ciencias de la educación o en pedagogía que se imparten en instituciones de educación superior públicas y privadas con prestigios, proyectos y orientaciones variados. En virtud de la autonomía propia del nivel superior, los planes de estudio de estos programas universitarios son distintos aunque compartan la denominación. Los objetivos curriculares y los perfiles de egreso se orientan con sentidos y énfasis diversos; las intenciones que respaldan los programas y los logros formativos son disímiles y las mallas curriculares se organizan con estructuras muy dispares. Comparten, sin embargo, algunas características esenciales que conviene tener presentes en el análisis que nos ocupa. Nos referimos, fundamentalmente, a un pronunciado alejamiento de la formación práctica que suponen las tareas directas de la enseñanza; un vacío de experiencias asociadas al ejercicio efectivo de la docencia, a las habilidades y adiestramientos puntuales relacionados con la intervención en espacios aúlicos y, desde luego, adolecen de la preparación específica en cuanto a los contenidos puntuales que requiere la transmisión en los niveles básicos del sistema educativo. En pocas palabras, la esencia de la formación inicial docente 
caracterizada tradicionalmente por el conocimiento de las materias a transmitir y el saber hacer propio de la impartición de clases y la intervención en el aula no juega ningún papel en el proyecto formativo que ofrecen estas licenciaturas.

$\mathrm{Al}$ margen de la heterogeneidad de proyectos formativos y resultados de egreso, puede ser fecundo focalizar el interés en una de estas licenciaturas, sólo como un recurso para ejemplificar el sentido de nuestras reflexiones.

\section{El caso de la licenciatura en Ciencias de la Educación de la UABC}

El análisis de algunos datos relacionados con el plan de estudios y de los resultados de los egresados de la licenciatura en Ciencias de la Educación de la Universidad Autónoma de Baja California (UABC) en los concursos de ingreso tiene utilidad para dar cuenta de cuestiones como el interés que puede despertar, o el acceso que puede presumirse tendrán los egresados de este tipo de carrera profesional al SPD.

La licenciatura en Ciencias de la Educación se ofrece en las facultades de Ciencias Humanas (FCH) (Mexicali) y de Ciencias Administrativas y Sociales (FCAyS) (Ensenada) de la UABC. En Mexicali, esta licenciatura se imparte, desde 1978, en la Escuela de Ciencias de la Educación, unidad académica que se convirtió en Facultad de Ciencias Humanas en 1993. En Ensenada, la licenciatura en Ciencias de la Educación se ofrece, desde su origen, en 2003, en la Facultad de Ciencias Administrativas y Sociales (UABC, 2012).

De acuerdo con el plan de estudios vigente, el licenciado en Ciencias de la Educación es un: "profesional que integra en su formación el estudio, reflexión y acción sobre la realidad educativa, $[\ldots$ y da] sustento al quehacer educativo en su universalidad, con el propósito de contribuir al desarrollo e impulso de estrategias para la solución de problemas integrando la identificación de áreas de oportunidad en los distintos ámbitos educativos" (UABC, 2012: 86).
El plan de estudios establece que el egresado de la licenciatura en Ciencias de la Educación es competente para:

- Producir e instrumentar estrategias educativas basadas en modelos convencionales, no convencionales y alternativos de formación requeridos para apoyar procesos formativos con un alto sentido ético, científico y humanista.

- Diseñar, instrumentar y evaluar planes y programas de estudio convencional y no convencional, mediando procedimientos innovadores del diseño curricular, tendientes a elevar la calidad educativa con actitud responsable y propositiva.

- Formular y evaluar procesos de gestión en instituciones educativas del país y del entorno regional utilizando modelos funcionales aplicables en la planeación y administración con el fin de lograr la mejora continua, así como la eficientización de los recursos en apego a la normatividad y principios institucionales, con actitud colaborativa.

- Desarrollar estudios relacionados a través de abordajes cuantitativos o cualitativos y mixtos con el objeto de aportar nuevas visiones o soluciones a las problemáticas educativas, con actitud crítica y proactiva (UABC, 2012: 86-87).

Desde las condiciones declaradas del plan de estudios, la intención de la licenciatura es la de formar profesionales de la educación, específicamente, en los campos de la didáctica general, del desarrollo curricular general, de la formación de profesores y la gestión de instituciones educativas; y se propone ofrecer bases para la iniciación en la investigación educativa. Es evidente que el plan de estudios de esta licenciatura no es en absoluto equivalente al que proyecta la formación inicial de docentes de preescolar y primaria que se imparte en la escuela normal 
ya que se trata de una licenciatura con un perfil profesional distinto.

\section{Resultados de los egresados de Ciencias de la Educación en los concursos de los ciclos escolares 2014-2015 y 2015-2016}

A fin de realizar este estudio, el Sistema Educativo Estatal (SEE) de Baja California proporcionó información general de los resultados de los egresados de la licenciatura de Ciencias de la Educación (de la FCH y de la FCAyS) en las convocatorias públicas abiertas para concursar por el ingreso al SPD de los ciclos escolares 2014-2015 y 2015-2016. Los datos obtenidos permiten establecer cuatro líneas de análisis:

1. Aspirantes egresados de Ciencias de la Educación de acuerdo con el profesiograma de la convocatoria pública y abierta de los concursos 2014-2015 y 2015-2016.

2. Resultados generales de los aspirantes de Ciencias de la Educación por facultades y concurso.

3. Resultados de los aspirantes de Ciencias de la Educación por facultades, concurso y niveles de desempeño.

4. Resultados de los aspirantes de Ciencias de la Educación por facultades, concurso y grupo de desempeño.

\section{Aspirantes de Ciencias de la Educación de acuerdo con el profesiograma de la convocatoria pública y abierta de los concursos 2014-2015 y 2015-2016}

En cumplimiento de lo dispuesto en el artículo 21 transitorio de la LGSPD, se publicaron dos convocatorias de concursos de oposición para los ciclos 2014-2015 y 2015-2016: la convocatoria para egresados de escuelas normales y la convocatoria pública y abierta. Este segundo tipo permitió participar a los egresados de las IES del país, públicas y particulares, en el concurso de ingresoal SPD como docente o técnico docente en los diferentes niveles y modalidades educativas.

Si bien abrir esta convocatoria era opcional para las entidades federativas, el SEE de Baja California decidió publicarla en los dos concursos. Un apartado de la convocatoria incluye una lista de las carreras seleccionadas para aspirar a ingresar al servicio en los distintos niveles y modalidades educativas. Esta lista se conoce como "profesiograma".

En el profesiograma de la convocatoria de 20142015, se encontró que la carrera de Ciencias de la Educación estaba aceptada en ocho niveles, modalidades y/o asignaturas, la carrera de Pedagogía en 17 y la carrera de Educación en 12. En la convocatoria 2015-2016, Ciencias de la Educación se aceptaba en seis niveles, modalidades y/o asignaturas, Pedagogía en 10 y Educación en nueve (Gobierno del Estado de Baja California, 2014; Gobierno del Estado de Baja California, 2015).

En las dos convocatorias analizadas no se indica: 1) el criterio utilizado para definir los perfiles profesionales seleccionados para determinadas asignaturas o las razones por las cuales en algunos casos se seleccionó a tres, dos o sólo una de ellas (por ejemplo, para el caso de educación preescolar se consideró la carrera de Ciencias de la Educación y Pedagogía, pero para educación primaria, se incluyeron las licenciaturas en Educación y Pedagogía); y 2) los criterios seguidos para modificar la lista de carreras de un ciclo escolar al otro. Se pudo observar, además, que la carrera de Ciencias de la Educación, en comparación con otras carreras afines, es la que tiene menos opciones de participación en los concursos 2014-2015 y 2015-2016.

Los egresados de Ciencias de la Educación de la UABC se registraron a los concursos de oposición en el marco de estas dos convocatorias públicas. La tabla 1 incluye la distribución de los sustentantes que se presentaron a ambos concursos por nivel educativo o modalidad del campus Mexicali y del campus Ensenada. 
En el ciclo escolar 2014-2015 concursó un total de 211 aspirantes egresados de Ciencias de la Educación (144 de la FCH y 67 de la FCAyS); en 2015-2016, la cifra se redujo a 154 aspirantes (95 de la FCH y 59 de la FGAyS). La disminución es explicable por la contracción de las opciones establecidas en el profesiograma establecido para el ingreso al SPD. La diferencia en el número de aspirantes por facultad puede obedecer al tamaño de la matrícula en cada campus. El promedio de egresados por generación de la FCAyS en las últimas cinco generaciones es de 28 alumnos, mientras que en la FCH es de 51 .
No existe una clara relación entre el profesiograma y los aspirantes registrados en los diferentes niveles, modalidades y asignaturas en los concursos de ingreso. La convocatoria de 2014-2015 permitió que los egresados de Ciencias de la Educación se presentaran al concurso de ingreso en educación secundaria en las materias de Formación Cívica y Ética, y Geografía. Esto podría explicar el alto número de aspirantes que se registraron en ambas materias. Sin embargo, a pesar de que en el profesiograma de la convocatoria 2015-2016 dicha carrera no estaba indicada para estas asignaturas,

Tabla 1. Distribución de frecuencias y porcentajes de los sustentantes egresados de Ciencias de la Educación que se presentaron en los concursos 2014-2015 y 2015-2016

\begin{tabular}{|c|c|c|c|c|c|c|c|c|}
\hline \multirow{3}{*}{ Nivel educativo/Modalidad } & \multicolumn{4}{|c|}{ Campus Mexicali FCH } & \multicolumn{4}{|c|}{ Campus Ensenada FCAyS } \\
\hline & \multicolumn{2}{|c|}{ 2014-2015 } & \multicolumn{2}{|c|}{ 2015-2016 } & \multicolumn{2}{|c|}{ 2014-2015 } & \multicolumn{2}{|c|}{ 2015-2016 } \\
\hline & $n$ & $\%$ & $n$ & $\%$ & $n$ & $\%$ & $n$ & $\%$ \\
\hline Educación especial & & & 1 & 1 & 1 & 1 & 8 & 14 \\
\hline Educación preescolar & 5 & 3 & 11 & 12 & & & 12 & 20 \\
\hline Educación primaria & 2 & 1 & 31 & 33 & 2 & 3 & 9 & 15 \\
\hline \multicolumn{9}{|l|}{ Educación secundaria: } \\
\hline Artes & & & 1 & 1 & & & & \\
\hline Biología & 2 & 1 & & & 1 & 1 & & \\
\hline Educación tecnológica & 1 & 1 & & & & & & \\
\hline Español & 1 & 1 & 1 & 1 & 1 & 1 & & \\
\hline Formación cívica y ética & 79 & 55 & 41 & 43 & 35 & 52 & 3 & 5 \\
\hline Geografía & 47 & 33 & 2 & 2 & 21 & 31 & 1 & 2 \\
\hline Historia & 4 & 3 & 1 & 1 & 3 & 4 & 2 & 3 \\
\hline Telesecundaria & 3 & 2 & 6 & 6 & 2 & 3 & 23 & 39 \\
\hline Técnico docente & & & & & 1 & 1 & 1 & 2 \\
\hline Totales & 144 & 100 & 95 & 100 & 67 & 100 & 59 & 100 \\
\hline
\end{tabular}

Fuente: Dirección de Evaluación. Sistema Educativo Estatal de Baja California. 
se registraron aspirantes al concurso, principalmente, en el municipio de Mexicali, si bien en menor número. Los porcentajes más altos de egresados del campus Mexicali que se presentaron al concurso 2015-2016 se concentran en Educación Primaria $(33 \%)$ y Formación Cívica y Ética $(43 \%)$ a pesar de que, para ninguna de ellas, se establece a la licenciatura en Ciencias de la Educación como uno de los perfiles considerados para este nivel y asignatura. Se observó, además, un aumento considerable de sustentantes egresados del campus Ensenada que participaron en el concurso 2015-2016 para ingresar a Telesecundaria, siendo que en la convocatoria estatal únicamente incluye para esa modalidad a las licenciaturas en Pedagogía y Educación.

Para el segundo concurso (2015-2016), la licenciatura en Ciencias de la Educación fue añadida como potencial aspirante a la modalidad de Educación Especial, en todas sus áreas (Auditiva y Lenguaje, Intelectual, Motriz, Psicología Educativa y Visual). Esto dio lugar al incremento de participantes, egresados del campus Ensenada, en el concurso de ingreso a esa modalidad. Las cifras crecieron del $1 \%$ al $14 \%$. No obstante, esta posibilidad no impactó en las aspiraciones de los egresados del campus Mexicali; de los que aplicaron al concurso sólo el 1\% se concentró en estas áreas.

\section{Resultados generales del concurso de los egresados en Ciencias de la} Educación por facultades y ciclo escolar

En la tabla 2 se presentan los resultados nacionales y estatales obtenidos en los concursos de ingreso 2014-2015 y 2015-2016 de la convocatoria de escuelas normales y de la convocatoria pública y abierta.

La tabla muestra que los porcentajes de resultados de aspirantes idóneos son mayores en el concurso 2015-2016 que en su predecesor 2014-2015, tanto para el ámbito nacional como para el estatal. Se observa, además, que los porcentajes de aspirantes idóneos egresados de escuelas normales son más altos que los de la convocatoria pública y abierta, en el ámbito nacional y estatal, en ambas convocatorias. Hay, en promedio, 12 puntos porcentuales de diferencia entre los resultados de los egresados de la convocatoria de escuelas normales y los de los egresados de la convocatoria pública y abierta.

Los resultados de los egresados de la licenciatura en Ciencias de la Educación de la UABC de ambos campus se presentan en la tabla 3 .

Tabla 2. Resultados generales de los sustentantes de los concursos de educación básica por tipo de convocatoria en el ámbito nacional y en Baja California. Concursos de ingreso 2014-2015 y 2015-2016

\begin{tabular}{|c|c|c|c|c|c|}
\hline \multicolumn{2}{|c|}{$\begin{array}{c}\text { Resultado general del concurso de } \\
\text { ingreso }\end{array}$} & \multicolumn{2}{c|}{$\begin{array}{c}\text { Resultados de la convocatoria } \\
\text { egresados de escuelas normales }\end{array}$} & \multicolumn{2}{c|}{$\begin{array}{c}\text { Resultados de la convocatoria } \\
\text { pública y abierta }\end{array}$} \\
\hline Idóneo (\%) & No idóneo (\%) & Idóneo (\%) & No idóneo (\%) & Idóneo (\%) idóneo (\%) \\
\hline 39.0 & 61.0 & 44.5 & 55.5 & 33.0 & 44.2 \\
\hline 52.0 & 48.0 & 59.0 & 41.0 & 42.0 & 55.8 \\
\hline 56.0 & 44.0 & 57.0 & 43.0 & 58.0 \\
\hline
\end{tabular}

Nota: adaptado de "Concurso de Oposición para el Ingreso a la Educación Básica ciclo escolar 2014-2015. Estadísticas del concurso" Coordinación Nacional del Servicio Profesional Docente (2014 y 2015a).

Recuperado de $h$ ttp://servicioprofesionaldocente.sep.gob.mx/ba/ingreso_historico_2014/estadisticas_concurso_a/ y de $h$ ttp://servicioprofesionaldocente.sep.gob.mx/ba/ingreso/estadisticas_concurso_en/ 
Al igual que la tendencia nacional en los tres casos arriba mencionados, los resultados de los egresados del campus Mexicali y Ensenada mejoraron sustancialmente en el concurso 2015-2016. En la tabla 3 puede observarse que el porcentaje de idóneos de la FGH pasó de $44 \%$ en 2014 a $63 \%$ de idóneos en 2015. Por su parte, la FGAyS tuvo $52 \%$ de aspirantes idóenos en 2014 y 58\% en 2015. No se conocen, sin embargo, documentos oficiales —ni de la Coordinación Nacional del Servicio Profesional Docente, ni del INEE - que ofrezcan explicación a la evidente mejoría de los resultados de todos estos grupos. ${ }^{5}$

Para los fines de este trabajo, haremos una revisión particular del resultado de estos aspirantes en el concurso para preescolar y primaria. El mayor número de aspirantes al nivel primaria egresó del

Tabla 3. Resultados de los licenciados en Ciencias de la Educación de la FCH (campus Mexicali) y de la FCAyS que presentaron concurso de ingreso al SPD

\begin{tabular}{|c|c|c|c|c|c|c|c|c|}
\hline \multirow{3}{*}{$\begin{array}{c}\text { Nivel educativo/ } \\
\text { Modalidad }\end{array}$} & \multicolumn{4}{|c|}{ Campus Mexicali FCH } & \multicolumn{4}{|c|}{ Campus Ensenada FCAyS } \\
\hline & \multicolumn{2}{|c|}{ 2014-2015 } & \multicolumn{2}{|c|}{ 2015-2016 } & \multicolumn{2}{|c|}{ 2014-2015 } & \multicolumn{2}{|c|}{ 2015-2016 } \\
\hline & Idóneo & No idóneo & Idóneo & No idóneo & Idóneo & No idóneo & Idóneo & No idóneo \\
\hline Educación especial & & & 1 & & & 1 & 4 & 4 \\
\hline Educación preescolar & 1 & 4 & 6 & 5 & & & 4 & 8 \\
\hline Educación primaria & & 2 & 19 & 12 & & 2 & 6 & 3 \\
\hline \multicolumn{9}{|l|}{ Educación secundaria: } \\
\hline Artes & & & & 1 & & & & \\
\hline Biología & & 2 & & & 1 & & & \\
\hline Educación tecnológica & & 1 & & & & & & \\
\hline Español & 1 & & 1 & & 1 & & & \\
\hline Formación cívica y ética & 36 & 43 & 28 & 13 & 16 & 19 & 2 & 1 \\
\hline Geografía & 24 & 23 & 1 & 1 & 13 & 8 & & 1 \\
\hline Historia & 1 & 3 & 1 & & 1 & 2 & 2 & \\
\hline Telesecundaria & 1 & 2 & 3 & 3 & 2 & & 15 & 8 \\
\hline Técnico docente & & & & & 1 & & 1 & \\
\hline Totales por rubro & 64 & 80 & 60 & 35 & 35 & 32 & 34 & 25 \\
\hline Porcentajes por rubro & $44 \%$ & $56 \%$ & $63 \%$ & $37 \%$ & $52 \%$ & $48 \%$ & $58 \%$ & $42 \%$ \\
\hline Total por año & \multicolumn{2}{|c|}{144} & \multicolumn{2}{|c|}{95} & \multicolumn{2}{|c|}{67} & \multicolumn{2}{|c|}{59} \\
\hline
\end{tabular}

Fuente: Dirección de Evaluación. Sistema Educativo Estatal de Baja California.

${ }^{5}$ Este tema requiere de un análisis particular. Es importante conocer a qué se atribuye la mejora en los resultados de la segunda convocatoria. Consideramos esta cuestión no puede quedar fuera de la agenda pendiente del impacto del SPD en la tarea educativa. 
campus Mexicali (31). De estos aspirantes, 19 (61\%) fueron idóneos y 12 (39\%) no idóneos. En cuanto a preescolar, se registraron 11 egresados del campus Mexicali y 12 del campus Ensenada. Los resultados fueron ligeramente mejores en el campus Mexicali. Aproximadamente la mitad resultó idóneo. En el caso de Ensenada, una tercera parte fue idóneo.

\section{Resultados de los aspirantes por facultades, concurso y niveles de desempeño}

El análisis de los resultados de los concursos de ingreso por niveles permite profundizar en la observación del desempeño de los egresados de la licenciatura en Ciencias de la Educación de la UABC. A tal fin coadyuvan, también, la descripción - si bien, breve - de las etapas de la evaluación, el señalamiento de los niveles de desempeño del examen y los criterios con los que se integraron los grupos de desempeño.

La evaluación para el ingreso se compone de dos exámenes: el Examen nacional de conocimientos y habilidades para la práctica docente (ENCHPD) (que comprende las dimensiones 1 y 2 de los PPI) y el Examen nacional de habilidades intelectuales y responsabilidades ético-profesionales (ENHIRE) (que integra las dimensiones 3, 4 y 5 de los PPI) (SEPCNSPD, 2015). Los resultados de cada examen se agrupan en tres categorías de desempeño: Niveles I, II y III, de acuerdo con las definiciones que se incluyen en la tabla 4.

La tabla 5 presenta los resultados de los sustentantes egresados de la licenciatura en Ciencias de la Educación por nivel de desempeño por facultad y por concurso en los dos exámenes aplicados.

Los egresados de Ciencias de la Educación parecen tener un resultado similar en ambos exámenes. En 2014, los sustentantes tuvieron un mejor desempeño en el ENCHPD. Ambas facultades tuvieron un porcentaje muy alto de niveles 1 (insuficientes) en el ENHIRE y un porcentaje mayor de suficientes en el ENCHPD. En 2015 hubo una mejora en los resultados. Bajó el porcentaje de insuficientes en el ENHIRE y en ambas facultades bajó el número de suficientes en el ENCHPD. Es notorio que, en 2015, aumentaron los porcentajes de sustentantes con nivel III en ambos exámenes. Es posible observar que en la convocatoria 2015, el incremento de los porcentajes puede indicar movilidad del nivel II al nivel III en el caso del ENCHPD y del nivel I al nivel III en el caso del ENHIRE. Es posible decir que, en los dos exámenes, en ambas facultades hubo una mejora en el porcentaje de sustentantes con desempeño destacado en la segunda convocatoria.

\section{Resultados de los aspirantes por facultades, concurso y grupo de desempeño}

Los grupos de desempeño se conforman de acuerdo con la combinación de los resultados alcanzados en los instrumentos considerados en el proceso de evaluación. Para el caso de la evaluación con dos exámenes, se conformaron tres grupos de desempeño: A, B y C. Los sustentantes que se incluyen en el grupo A son los que se encuentran en los primeros lugares en la lista de prelación. En la tabla siguiente se presentan los criterios para formar parte de cada grupo.

Los resultados de los egresados de Ciencias de la Educación por campus se presentan en la tabla 7. Puede observarse que, en general, el mayor porcentaje de los aspirantes con resultados idóneos se ubican en el nivel de desempeño más bajo, nivel C. Dicho porcentaje de desempeño $\mathrm{C}$ disminuye en el ciclo escolar 2015-2016 y aumenta para el caso de los idóneos con niveles A. Para el caso específico de preescolar, en los dos campus los idóneos se ubicaron en niveles B y C. Para el caso de primaria, en los dos campus, en 2015-2016, los idóneos nivel A fueron alrededor del $30 \%$, por lo que su probabilidad de ingresar al servicio se incrementó. 
Tabla 4. Definición de los niveles de desempeño

\begin{tabular}{|c|l|}
\hline Nivel & \multicolumn{1}{c|}{ Definición } \\
\hline Nivel I & $\begin{array}{l}\text { Significa un dominio insuficiente de los conocimientos y habilidades que se consideran } \\
\text { indispensables para un adecuado desempeño docente. }\end{array}$ \\
\hline Nivel II & $\begin{array}{l}\text { El sustentante muestra un dominio suficiente y organizado de los conocimientos y } \\
\text { habilidades que se juzgan indispensables para un adecuado desempeño docente. }\end{array}$ \\
\hline Nivel III & $\begin{array}{l}\text { Significa que el sustentante, además de mostrar un dominio suficiente y organizado de } \\
\text { los conocimientos y habilidades contemplados en el instrumento, demuestra una amplia } \\
\text { capacidad para utilizarlos en una diversidad de situaciones didácticas. }\end{array}$ \\
\hline
\end{tabular}

Fuente: CNSPD (2015b). Recuperado de http://servicioprofesionaldocente.sep.gob.mx/ba/ingreso/criterios_basicos/

\section{Tabla 5. Resultados de los sustentantes egresados de la licenciatura en Ciencias de la Educación por nivel de desempeño en los dos exámenes de ingreso dividido por campus en las convocatorias 2014 y 2015}

\begin{tabular}{|l|r|r|r|r|r|r|r|r|}
\cline { 2 - 9 } & \multicolumn{4}{c|}{ Convocatoria 2014 } & \multicolumn{4}{c|}{ Convocatoria 2015 } \\
\cline { 2 - 9 } & \multicolumn{2}{|c|}{ FCH } & \multicolumn{2}{c|}{ FCAyS } & \multicolumn{2}{c|}{ FCH } & \multicolumn{2}{c|}{ FCAYS } \\
\cline { 2 - 9 } & ENHCPD & ENHIRE & ENHCPD & ENHIRE & ENHCPD & ENHIRE & ENHCPD & ENHIRE \\
\hline Nivel I & 31.94 & 45.83 & 23.88 & 40.3 & 25.26 & 28.42 & 30.51 & 23.73 \\
\hline Nivel II & 57.64 & 47.92 & 68.66 & 46.27 & 43.16 & 45.26 & 47.46 & 55.93 \\
\hline Nivel III & 10.42 & 6.25 & 7.46 & 13.43 & 31.58 & 26.32 & 22.03 & 20.34 \\
\hline
\end{tabular}

Fuente: Dirección de Evaluación. Sistema Educativo Estatal de Baja California.

Tabla 6. Criterios para formar parte de un grupo de desempeño

\begin{tabular}{|c|c|}
\hline $\begin{array}{c}\text { Grupos de } \\
\text { desempeño }\end{array}$ & Descripción \\
\hline A & En los dos exámenes obtuvo el nivel de desempeño III \\
\hline B & En un examen obtuvo el nivel de desempeño III y en el otro el nivel de desempeño II \\
\hline C & En los dos exámenes obtuvo el nivel de desempeño II \\
\hline
\end{tabular}

Fuente: CNSPD (2015b). Recuperado de http://servicioprofesionaldocente.sep.gob.mx/ba/ingreso/criterios_basicos/ 
Tabla 7. Distribución de resultados del concurso de nuevo ingreso de los licenciados en Ciencias de la Educación de la FCH y de la FCAys que resultaron idóneos por convocatoria

\begin{tabular}{|c|c|c|c|c|c|c|c|c|}
\hline \multirow[b]{2}{*}{$\mathrm{FCH}$} & \multicolumn{4}{|c|}{ 2014-2015 } & \multicolumn{4}{|c|}{$2015-2016$} \\
\hline & Idóneo & $\boldsymbol{A}$ & B & c & Idóneo & $\boldsymbol{A}$ & $B$ & $c$ \\
\hline Totales de los aspirantes: & 64 & 2 & 17 & 45 & 60 & 15 & 25 & 20 \\
\hline Totales en porcentaje: & 100 & 3 & 27 & 70 & 100 & 25 & 42 & 33 \\
\hline FCAyS & Idóneo & $\boldsymbol{A}$ & $B$ & $c$ & Idóneo & $\boldsymbol{A}$ & $B$ & $c$ \\
\hline Totales de los aspirantes: & 35 & 2 & 9 & 24 & 34 & 8 & 8 & 18 \\
\hline Totales en porcentaje: & 100 & 6 & 26 & 68 & 100 & 24 & 24 & 52 \\
\hline
\end{tabular}

Fuente: Dirección de Evaluación. Sistema Educativo Estatal de Baja California.

\section{Discusión}

\section{Meritocracia en el SPD}

La selección de los individuos para ocupar posiciones sociales y profesionales con base en el mérito - y no por condiciones marcadas por el azar natural o social, como el sexo, la etnia, el origen social o, incluso, por las redes de relaciones o vínculos con grupos de autoridad y de poder - es parte de una larga tradición en Occidente (Puyol, 2010: 101). Se trata de una tradición que hunde sus raíces en la consideración de la igualdad de derechos de todos en la competencia social. En teoría, asociamos el mérito con una de las condiciones que hacen posible una sociedad igualitaria y la meritocracia podría ser enjuiciada como un sistema de repartos que premia el talento y el esfuerzo personales, antes que la diferenciación en los repartos sociales de riqueza y bienestar.

Sin desestimar la importancia que el examen sociológico, los estudios estadísticos y las elaboraciones teóricas de la ciencia y la filosofía políticas han desarrollado para sostener la inexactitud de la valoración desmedida que nuestra sociedad hace del mérito y la meritocracia ${ }^{6}$ como factores de transformación de las estructuras sociales injustas, su incorporación al esquema de ingreso, promoción, permanencia y reconocimiento en la carrera docente podría ser reconocida, al menos, como la instauración de un nuevo modelo. No obstante, y a pesar del lugar casi indiscutido que el mérito tiene entre los valores de las sociedades modernas y el de la meritocracia como un modelo social extendido en las tradiciones de organización occidental como sistema de distribución de plazas profesionales y puestos sociales, su aplicación no queda siempre exenta de objeciones. El caso de la reforma educativa de 2013, por su parte, no ha logrado superar muchas dificultades - añadidas a los defectos propios del modelo- que tendrían que ser sofisticadamente atendidas en la consideración del mérito de cara a la incorporación de la carrera docente.

\footnotetext{
${ }^{6} \mathrm{Si}$ bien, para los propósitos de este artículo, no es necesario traer a colación los sistemas analíticos y explicativos que desde la historia, la sociología, la filosofía y la pedagogía se han ofrecido para dar cuenta de los mecanismos que la meritocracia - como una forma de "aristocracia del talento"(Puyol) - impone a la conservación y reproducción de las jerarquías sociales, no está de más señalar que más que un compromiso con la igualdad, el sistema meritocrático favorece la eficiencia y, sobre todo, la diferenciación. Cf. Bourdieu y Passeron (1996); Baudelot y Leclerc (2008); Dubet (2011); Puyol (2010) y Tedesco (2012), entre otros.
} 
Los resultados de la participación de egresados de las licenciaturas en Ciencias de la Educación de la UABC en el ejercicio promovido en el estado de Baja California bajo el esquema dispuesto en el artículo 21 transitorio de la LGSPD para los concursos de ingreso 2014-2015 y 2015-2016 podrían fungir como indicadores - obviamente, entre otros - para estudiar los efectos y el significado que atribuye el registro meritocrático de la reforma educativa en el tránsito para ocupar las plazas magisteriales en nuestro país. No proveen un panorama completo del tipo de resultados esperables a futuro o a nivel nacional, pero sí aportan elementos que permiten develar algunos de los rasgos de la semi-oculta transformación del modo de entender el tipo de perfil profesional y el carácter de idoneidad que la condición meritocrática de la reforma supone implicada en el ejercicio de la tarea docente.

Los resultados no son despreciables. No sólo es evidente que hay un interés destacado, por parte de los egresados de esas licenciaturas, en incorporarse al SPD; también hay datos relevantes que muestran que buena parte de ellos logran ser idóneos y que, progresivamente, lo han logrado con mejores resultados en la edición 2015-2016 del concurso. No son despreciables, sobre todo, si la interpretación de tales resultados observa las radicales diferencias que existen entre los programas de estudio de las licenciaturas en que se han formado los licenciados en Ciencias de la Educación y los requerimientos reconocidos, de facto, en las tareas de transmisión y educación escolarizada. Resulta, por ello, indispensable detenerse a analizar la compleja trama de pre-supuestos, creencias, ejecuciones habituales fincadas en la rutina y tradiciones asentadas en el registro de la comprensión del estudio, el desarrollo de los proyectos formativos y la práctica en torno a los problemas educativos, entre ellos, los de las tareas propias de la enseñanza y los factores asociados a la escolarización.

¿Qué hay en juego en la determinación de estos resultados? Y ¿qué consideraciones teóricas tendrían qué hacerse para analizar las razones y los efectos de tales resultados a corto, mediano y largo plazos?

\section{La formación de profesores y la formación de profesionistas de la educación}

Uno de los temas a considerar, en esta línea de reflexión, tiene que ocuparse de las distancias formativas establecidas en los perfiles de quienes han cursado las licenciaturas destinadas a formar profesionales de la enseñanza (en las escuelas normales); y los de quienes han cursado las licenciaturas en Educación, en Ciencias de la Educación o en Pedagogía (en el sistema de universidades e IES). Axiomáticamente es admitido que la formación magisterial asume como indispensable: la adquisición de las habilidades, las disposiciones y el conocimiento de un fondo cultural común, que han de desplegarse en la transmisión de los contenidos establecidos en los programas del nivel básico y en la articulación de la injerencia educativa en la especificidad del espacio escolar. Nos referimos, expresamente, a ese conjunto de capacidades cuya aplicación tiene su destino directo en el aula, bajo el marco de la relación educativa escolarizada. Es ahí, en la escuela, donde el educador - como representante autorizado del mundo de los adultos - interactúa con los jóvenes, con los "nuevos" para mostrar y transmitir el conocimiento que posee la especie humana sobre "nuestro mundo", y abrir, a los "nuevos", la posibilidad de "renovarlo", de reinventar "lo que nos es común" (Arendt, 1968: 301). ${ }^{7}$

\footnotetext{
${ }^{7}$ A fin de conocer de qué manera se define este perfil en el caso del profesor mexicano, se recomienda revisar las competencias genéricas y profesionales del Plan de estudios 2012 de la Licenciatura en Educación Primaria. Disponible en http://www.dgespe.sep.gob.mx/ reforma_curricular/planes/lepri/plan_de_estudios/perfil_egreso
} 
Históricamente, la comprensión respecto del peculiar carácter de la pedagogía, la educación o la ciencia de la educación, en el sentido de ser una disciplina teórico-práctica y del tipo particular de vínculos que, en su seno, sostienen la experiencia y la abstracción, ${ }^{8}$ ha cursado por momentos de profunda confusión; no obstante, su dimensión normativa y su carácter científico se encuentran ya hoy bien delineados e iluminan los senderos de la formación de sus profesionales.

No es éste el lugar para detenernos a reconstruir las incontables dicotomías que han puesto en jaque el carácter mixto de la estructura conceptual de la pedagogía y las peculiaridades de su vínculo con el ejercicio práctico de la enseñanza escolarizada o de otras prácticas educativas. ${ }^{9}$ Lo que importa mostrar es el hecho de que la construcción y reconstrucción de teorías pedagógicas exige de una desviación, eventualmente pronunciada, de las operaciones prácticas puntuales.

No intentamos sostener la indefendible separación tajante y desvinculante del ejercicio intelectual y el trabajo práctico, como se desprendería de una mirada burda de trabajo dividido. Pretendemos, más bien, subrayar que el desarrollo del quehacer pedagógico supone un registro temporal, derivado de las condiciones lógicas y empíricas del ejercicio disciplinario, que ha de enfrentar a la extendida y natural inclinación a probar que los resultados científicos, y los modos de estudiar los asuntos educativos, puedan transformarse — sin más - en reglas y modelos prácticos.

En La ciencia de la educación, John Dewey intentó develar la condición de complejidad que la administración e instrucción escolares implican por encima de los factores contenidos en los resultados científicos derivados de las disciplinas que informan al hacer pedagógico.
De acuerdo con él, "Ninguna conclusión de la investigación científica puede convertirse en una regla inmediata del arte educativo. Pues no existe una práctica educativa que no contenga otras muchas condiciones y factores que estén incluidos en el hallazgo científico" (Dewey, 1964: 23).

La intención del pragmatista norteamericano no era, por cierto, la de negar la utilidad práctica de los descubrimientos científicos; tampoco, por supuesto, la de establecer ninguna especie de jerarquización epistémica de las prácticas de la escolarización por encima de las actividades de generación de conocimiento. Su pretensión cursaba, más bien, por el intento de indicar que - una vez reconocidos los insolubles vínculos entre la teoría y la práctica educativas - se hacía necesario distinguir a una de otra sin elevar sus diferencias a la categoría abismal de una dicotomía irreconciliable.

Dewey insistía en el potencial normativo y práctico de los hallazgos intelectuales. "La teoría [sostenía en una conocida y lúcida frase] es la más práctica de todas las cosas" (1964: 21). En mucho, debemos a él la convicción, ampliamente aceptada, respecto de que no existe práctica - al menos no práctica inteligente - sin la teoría que la respalde (Dewey, 1998). Es, sin embargo, significativo el sentido en que subraya la necesidad de la distinción entre los hallazgos desarrollados por la ciencia de la educación (o por las ciencias fuente que le nutren) y su potencial de traslado al ámbito de las prácticas educativas.

Lo que de acuerdo con él modifica - en el práctico- el conocimiento de los hallazgos intelectuales, de las leyes y sistemas teóricos, es la conexión de hallazgos de la ciencia que mutuamente se iluminan para transformar el proceder específico en sus actos de intervención. Las aportaciones de los desarrollos

\footnotetext{
${ }^{8}$ Explicaciones extensas y claras sobre el carácter de este vínculo pueden encontrarse en obras como: Nohl (1952); Passmore (1983); Dewey (1964); Furlán y Pasillas (1999); Carr (2002), y otras.

${ }^{8}$ De ellas dan cuenta, entre otros, los recientes trabajos de autores que, como Eduardo Sota (en prensa), recorren sistemáticamente los modos de partición dicotómica y jerarquización que han hecho pasar el signo normativo de la ciencia de la educación o la pedagogía por reduccionismos que apuntan, bien al señalamiento puntual de instrumentos técnicos, o bien, a programas de producción de conocimiento desprendido de las prácticas de transmisión reconocidas como educativas.
} 
disciplinarios flexibilizan el hacer del docente en torno a sus posibilidades de elección de procedimientos, pues enriquecen sus juicios y reconstruyen su conducta en el tratamiento de las condiciones particulares que demandan su acción.

Los descubrimientos, orientaciones prácticas y encuentros de condiciones de generalización producidos por la disciplina de estudio de los fenómenos educativos no son cadenas de reglas de aplicación directa para el práctico, sino "instrumentos intelectuales" que indirectamente operan como medios para alterar la actitud mental de quien realiza los procedimientos empíricos (1964: 33).

Si Dewey tiene razón y, como sugiere, es posible hacer una distinción - a la vez radical y sutil -10 entre lo que suponen el conocimiento, las habilidades y las capacidades presupuestas en el desarrollo de la actividad destinada a la comprensión, construcción y reconstrucción de los sistemas intelectuales con que entendemos la labor del pedagogo y su aplicabilidad práctica a manos del ejercicio docente, parece razonable pensar que hay una suerte de vicio en la sentencia de la reforma educativa que supone equivalentes los proyectos formativos de las escuelas normales y los de las licenciaturas en Educación, en Ciencias de la Educación o en Pedagogía.

Es ésta la razón, o una de las razones, de que los curricula de los programas destinados a la formación de pedagogos o científicos de la educación difieran del que sostiene la formación del profesional de la docencia.

\section{La desacertada suposición de equivalencia de los dos tipos de programas formativos}

A la luz del tipo de servicios que presta la elaboración pedagógica al ejercicio de la práctica docente que hemos descrito, es posible suponer que lo que persiste tras la supuesta equivalencia de proyectos formativos es una mala comprensión de las habilidades y disposiciones que requiere el trabajo de los prácticos en los espacios escolares. Ello daría lugar a suponer que los resultados exitosos en los exámenes de ingreso obtenidos por los egresados de las licenciaturas en ciencias de la educación en el estado de Baja California pudiera contemplarse desde una óptica que, lejos de declararlos como capaces de desempeñarse eficientemente en las tareas de la enseñanza que tocan al SPD, expresaran - en realidad - la posesión de saberes disciplinarios susceptibles de ser traducidos en el campo específico de la intervención.

Pero la capacidad de realizar tal traducción de los saberes disciplinarios a procedimientos recomendables en contextos generalizables es justamente el señalamiento de que han recibido la formación pedagógica que se esperaba del programa de licenciatura que cursaron. De ahí a que pueda asegurarse su habilidad para las prácticas de intervención directa en el aula, queda una gran distancia.

De acuerdo con Eduardo Sota, la labor del pedagogo se encuentra en una posición intermedia entre los desempeños y compromisos prácticos y los discursos y desarrollos de la investigación científica. Sota se inclina por definir la labor del pedagogo como equivalente a la de un políglota capaz:

$$
\begin{aligned}
& \text { de traducir - no extrapolar - los resultados per- } \\
& \text { tinentes de las investigaciones educativas para } \\
& \text { diseñar dispositivos de intervención en función } \\
& \text { de los fines que lo animan respetando la lógi- } \\
& \text { ca de cada una de esas gramáticas a la vez de ser } \\
& \text { perceptivo de los sutiles vínculos que los interrela- } \\
& \text { cionan [...] el pedagogo debe asumir una función }
\end{aligned}
$$

\footnotetext{
${ }^{10}$ La sutileza de la distinción estriba, particularmente, en que las prácticas educativas son el principio y el fin de la tarea pedagógica. El objeto de estudio de la pedagogía, o la ciencia de la educación, es sólo y exclusivamente el de la práctica educativa. Son las prácticas de la educación las que ofrecen la materia de estudio, tanto como son ellas las que dan cuenta del valor de sus conclusiones. "Si el pedagogo se detiene a estudiar el trabajo docente — dicen Furlán y Pasillas (1999) — lo hace en la perspectiva de encontrar experiencias cuya generalización se estime recomendable o inconveniente. Lo hace para recomendar, que es su sino" (1999:10).
} 
mediadora-traductora entre cada uno de ellos pero a los fines de construir artefactos de intervención y transformación de la realidad, que es la dimensión praxeológica de su disciplina y donde finalmente se consuma su tarea (Sota, en prensa).

No obstante, la función del profesionista universitario capaz de realizar esta mediación-traductora, no diluye la indispensable distinción de los requerimientos para su ejecución y los que son propios para la conducta inteligente en el ejercicio del práctico, ni desarticula la especificidad de la actividad docente. Las acciones de transmisión y exposición a experiencias educativas que definen la función del maestro requieren de capacidades específicas y éstas se consiguen y desarrollan con la formación apropiada, con la intención deliberada y sistemática de su estimulación, de su habilitación, a partir de aproximaciones sucesivas de acercamiento a la práctica docente y a su ejercitación sostenida.

\section{Efectos, a corto plazo, de la equivalencia de los dos tipos de formación}

La disposición de la LGSPD en lo que se refiere a la consideración de los dos tipos de formación como equivalentes es relevante en el contexto que proyecta la escasez de maestros de educación básica (INEE, 2015a). La aplicación de la ley señalada parece tener dos efectos a muy corto plazo. Por un lado, los maestros se retirarán del servicio al cumplir los requisitos de ley y, por otro, la escuela normal ha dejado de ser atractiva para los egresados de nivel medio superior, dadas las nuevas condiciones laborales.

De acuerdo con los datos del INEE, la situación será grave en primaria y secundaria en el futuro inmediato (INEE, 2015a). Los egresados de las escuelas normales no serán suficientes para cubrir las plazas vacantes. En diez años - estima el INEE - el sistema de educación normalista "no podrá cubrir las demandas promedio de cada año originadas por las vacantes docentes" (INEE, 2015a: 49). Es decir, el número de egresados de normales, tanto para preescolar, como para primaria y secundaria, no será suficiente para cubrir los lugares que dejarán los pensionados potenciales. En los siguientes años, se prevé que habrá 48212 vacantes en primaria y el número de egresados normalistas será sólo de 9000 aproximadamente. Para el nivel secundario se estima un número aproximado a las 33606 vacantes y se estima que egresen sólo 8000 licenciados en educación secundaria de las normales superiores. Si se admite la amplia posibilidad de que no todos los que egresen cumplirán con el requisito de idoneidad, la situación podría ser crítica (INEE, 2015a).

Contrariamente a lo que la regulación normativa impone en atención a la mal asumida equivalencia de los perfiles profesionales del maestro normalista y el licenciado en Ciencias de la Educación o el pedagogo, el propio INEE reserva espacios para dudar de la fiabilidad de tal identificación. Pesa, eventualmente, en algunos de sus discursos, el reconocimiento de que se precisa de un profesional docente con disposiciones articuladas y exposición a experiencias de tratamiento de los fenómenos concretos de la práctica. Señala, ocasionalmente, que es necesario buscar mecanismos para "asegurar la calidad" de la oferta académica alterna a la escuela normal y "evitar que un posible déficit sea cubierto por docentes sin las calificaciones necesarias, contrario a lo concebido en la política educativa nacional" (INEE, 2015b: 50). En las Directrices para mejorar la formación inicial de los docentes de educación básica, el Instituto plantea crear un "Marco Común" de educación superior para la formación inicial de docentes. El propio Instituto sugiere que:

deberán abordarse los elementos clave del Marco Común (principios básicos, estructuras organizativas, objetivos, metas, procedimientos, criterios e incentivos para la colaboración) y sentarse las bases para el desarrollo de una política pública de articulación interinstitucional en el corto plazo 
considerando las ventajas comparativas de las escuelas normales y de las IES. A partir de ello, si bien deberán de respetarse los contextos y la identidad de las instituciones, se buscará disminuir las asimetrías organizacionales y de gestión existentes (INEE, 2015b: 24).

Considera el INEE que las asimetrías son estrictamente organizacionales y de gestión y que pueden anularse vía la integración de las escuelas normales y las demás IES en aras de conformar un sistema de formación inicial "pertinente y de calidad"; suponen que, con el auxilio de ese discurso, podrían desarticularse las condicionantes epistémicas que constituyen el corazón de las tareas magisteriales.

\section{Efectos, a largo plazo, de la equivalencia de los dos tipos de formación}

Se trata, no obstante, de un hito - sólo aparentemente administrativo y de gestión - que amenaza con proponer una re-significación de lo que es y hace un maestro de escuela y ello puede tener consecuencias graves a más largo plazo.

Lo que hay en la base del planteamiento es, en última instancia, una percepción de que la labor docente puede tener un carácter estrictamente instrumental. Sin embargo, los maestros transmiten, generan condiciones que hacen posible el aprendizaje, proyectan posibilidades de incorporación, de rechazo, de transformación, influyen, despliegan actividades que promueven el crecimiento, la concientización, la autonomía, el progreso; en una palabra: enseñan. Recurren para ello al diseño y aplicación de actividades y tecnologías que conocen y respaldan por la condición de ser sujetos de saber, y porque saben hacerlo.
De acuerdo con John Passmore, existe una condición lógica en la conceptualización de la enseñanza, sin cuyo asentamiento no puede ser entendida, a saber: la de que enseñar supone una relación triádica en donde el que enseña, debe enseñar algo a alguien. "Por cada $X$, si $X$ enseña, debe haber alguien a quien enseña y algo que enseña [...] El que 'él enseñe' funcione como un enunciado completo fomenta la creencia de que 'enseñar' es el nombre de una habilidad específica en la cual una persona es 'buena' o 'mala',' tal como nadar es el nombre de una habilidad en la cual una persona es buena o mala, nade donde nade y cuando nade"(1983: 36).

Lo que la condición lógica implicada en la relación triádica que determina el ser de la enseñanza es indicativa de las condiciones que hacen indispensable un tipo particular de formación para el ejercicio docente. Es decir, el bagaje sustantivo respecto de las materias de la enseñanza y las disposiciones que son necesarias para establecer la relación productiva que es necesaria con el contenido de la enseñanza y con los destinatarios de la actividad de enseñanza.

Aun en la versión rancieriana de un maestro ignorante, quien enseña, se arrogue o no el saber ignorado por el ignorante, sabe muy bien cómo hacer de ello un objeto de saber para el otro y en qué momento, bajo qué condiciones, y de acuerdo con qué procedimiento puede procurar la reducción de la ignorancia que convoca su intervención (Rancière, 1987 y 2008).

Queda claro que no basta con el saber específico de la materia objeto de la transmisión, como dice Diana Paredes, el contexto escolar impone que:

el educador no deba preocuparse por tener a la mano el saber a enseñar - ya que él mismo lo encana- sino que debe estar atento a las interacciones

\footnotetext{
${ }^{11}$ Este reconocimiento respecto de que la 'bondad' o 'maldad' del enseñante no pueda sostenerse al margen del contenido mismo de la enseñanza y de la especificidad de los destinatarios de la acción de quien enseña es un factor relevante para sostener una perspectiva crítica frente la aplicación de instrumentos de medición de las capacidades del desempeño docente. El estudio de validez y confiabilidad de los instrumentos que se utilizan en el concurso de ingreso y las medidas de presentación pública de sus resultados, son aún tareas pendientes para el INEE.
} 
que los jóvenes tengan con el conocimiento de que dispone...El educador debe considerar que para el experto, las materias tienen un carácter extensivo, están medianamente definidas y sus temas se encuentran interconectados, mientras que para el estudiante no presentan tales características. En ese sentido, su función es la de hacer mover a éste último hacia la dirección que el experto ya conoce y, simultáneamente, ser capaz de identificar las necesidades y capacidades que las nuevas generaciones presentan (Paredes, 2015: 211).

Este señalamiento inscribe, en la función del maestro, las condiciones epistémicas y operativas que contraponen la especificidad de su actividad versus la que es propia del profesionista de la educación (el científico de la educación o el pedagogo). Y de ello, las IES tienen plenas noticias. Parece impensable que, de la noche a la mañana, y en atención exclusivamente a la apertura de una potencial oferta del mercado laboral (como la que se desprenderá de los concursos diseñados al amparo del nuevo SPD), las universidades fuercen y transgredan el espíritu de las disciplinas y comprometan las condiciones lógicas y epistemológicas que suscriben sus proyectos de formación de profesionistas.

No negamos que pudiera resultar fértil que las licenciaturas en Pedagogía, en Educación o en Ciencias de la Educación, implicaran en su formación estrategias, mecanismos y condiciones que acercaran, al futuro profesionista, a saberes prácticos que hoy están sólo al alcance de quienes cursan por los programas de las escuelas normales. Pero la intención, en caso de que lo hicieran, no podría ser la de convertir a los pedagogos en maestros de escuela, sino la de perfeccionar sus herramientas de traducción y de mediación entre los saberes teóricos y los prácticos. En todo caso, la tarea de las IES que cobijan los programas de formación de profesionistas en educación, tendría que ser la de impulsar la crítica y señalar los peligros que la Reforma impone al articular - por la vía de una forzada homologación de perfiles profesionales - la re-significación del lugar y el papel del magisterio. 


\section{Referencias}

Arendt, H. (1968), "La crisis de la educación", en Entre el pasado y el futuro. Ocho ejercicios sobre la reflexión política, Barcelona, Ediciones Península, pp. 269-301.

Baudelot, C. y F. Leclerq (2008), Los efectos de la educación, Buenos Aires, Centro de Estudios Multidisciplinarios/ Del estante editorial.

Bourdieu, P. y J. Passeron (1996), La reproducción. Elementos para una teoría del sistema de enseñanza, México, Fontamara.

Carr, W. (2002), Una teoría para la educación. Hacia una investigación educativa crítica, Madrid, Morata.

Comité para la evaluación de programas de Pedagogía y Educación (CEPPE) (2015), "Programas acreditados", <http://www.ceppe.org.mx/acreditacion/programasacreditados/> [Consulta: enero de 2016].

Coordinación Nacional del Servicio Profesional Docente (GNSPD) (2014), "Concurso de Oposición para el Ingreso a la Educación Básica ciclo escolar 2014-2015. Estadísticas del concurso", http:// servicioprofesionaldocente.sep.gob.mx/ba/ingreso_ historico_2014/estadisticas_concurso_a/> [Consulta: enero de 2016].

CNSPD (2015a), "Concurso de Oposición para el Ingreso a la Educación Básica ciclo escolar 20152016. Estadísticas del concurso", <http:// servicioprofesionaldocente.sep.gob.mx/ba/ingreso/ estadisticas_concurso_en/> [Consulta: enero de 2016]. CNSPD (2015b), "Criterios básicos para calificar a los sustentates", <http://servicioprofesionaldocente.sep. gob.mx/ba/ingreso/criterios_basicos/> [Consulta: abril de 2016 ].

Cuenca, R. (2015), "Las carreras docentes en América Latina. La acción meritocrática para el desarrollo profesional", Santiago, Orealc/Unesco, <http://www. politicasdocentesalc.com/images/stories/Biblioteca/ Carrera_docente_Informe_FINAL_Guenca $\% 20$ 090315.pdf> [Consulta: enero de 2016].

Dewey, J. (1964), La ciencia de la educación, Buenos Aires, Losada.

Dewey, J. (1998), Democracia y educación, Madrid, Morata.
Diario Oficial de la Federación (DOF) (2013a), "Decreto por el que se reforman los artículos 3o. en sus fracciones III, VII y VIII; y 73, fracción XXV, y se adiciona un párrafo tercero, un inciso d) al párrafo segundo de la fracción II y una fracción IX al artículo 3o. de la Constitución Política de los Estados Unidos Mexicanos", <http://www.dof.gob.mx/nota_detalle. php? $\operatorname{codigo}=5288919 \&$ fecha $=26 / 02 / 2013>$ [Consulta: enero de 2016].

DOF (2013b), "Decreto por el que se expide la Ley General del Servicio Profesional Docente", <http:// www.dof.gob.mx/nota_detalle.php? codigo=53138 $43 \&$ fecha $=11 / 09 / 2013>$ [Consulta: septiembre de 2015].

Dubet, F. (2011), Repensar la justicia social. Contra el mito de la igualdad de oportunidades, Buenos Aires, Siglo XXI.

Furlán, A. y M. A. Pasillas (1999), "El campo pedagógico", en Notas sobre la pedagogía, México, UNAM.

Gobierno del Estado de Baja California (2014), "Concurso de Oposición para el Ingreso a la Educación Básica, ciclo escolar 2014-2015”, <http:// servicioprofesionaldocente.sep.gob.mx/content/ba/ docs/abierta/convocatorias/02_P.pdf > [Consulta: enero de 2016].

Gobierno del Estado de Baja California (2015), "Concurso de Oposición para el Ingreso a la Educación Básica, ciclo escolar 2015-2016", <http://servicioprofesionaldocente. sep.gob.mx/content/ba/docs/2015/ingreso/ convocatorias/abierta/ConvocatoriaBasicaAbierta_2. pdf $>$ [Consulta: enero de 2016].

Instituto Nacional para la Evaluación de la Educación (INEE) (2015a), Los docentes en México, México, SEP.

INEE (2015b), "Directrices para mejorar la formación inicial de los docentes de educación básica", México, INEE, <http://www.inee.edu.mx/images/ stories/2015/directrices/Directrices-0915.pdf> [Consulta: marzo de 2016].

Nohl, H. (1952), Teoría de la educación, Buenos Aires, Losada. Paredes, D. M. (2015), "Pautas para la formación de 
maestros desde la perspectiva deweyana", en Juan Ramos y otros (coords.), Deliberar con Fohn Dewey: ciencias sociales y educación, México, UPN, pp. 205-227.

Passmore, J. (1983), Filosofia de la enseñanza, México, Fondo de Cultura Económica.

Puyol, Á. (2010), El sueño de la igualdad de oportunidades. Crítica de la ideología meritocrática, Barcelona, Gedisa.

Rancière, J. (1987), El maestro ignorante. Cinco lecciones sobre la emancipación intelectual, Barcelona, Laertes.

Rancière, J. (2008), El espectador emancipado, Buenos Aires, Manantial.

Sandoval, E. (2009), "La inserción a la docencia. Aprender a ser maestro de secundaria en México", en Revista del curriculum y formación del profesorado, vol. 13, núm. 1, pp. 183-194.

Secretaría de Educación Pública (SEP) (2016), "Perfil, parámetros e indicadores para docentes y técnicos docentes. Concurso de oposición para el ingreso a la educación básica. Ciclo escolar 2016-2017”, <http:// servicioprofesionaldocente.sep.gob.mx/content/ba/
docs/2016/ingreso/PPI_INGRESO_BASICA_2016. pdf> [Consulta: marzo de 2016].

SEP-CNSPD (2015), "Etapas, aspectos, métodos e instrumentos. Proceso de evaluación para el ingreso a la educación básica", México, SEP, <http:// servicioprofesionaldocente.sep.gob.mx/content/ba/ docs/2015/ingreso/Etapas_aspectos_y_metodos_ Ingreso_15-N.pdf.> [Consulta: enero de 2016].

Sota, E. (en prensa), "Pedagogía", en A. M. Salmerón, B. F. Trujillo y otros (coords.), Diccionario Iberoamericano de Filosofia de la Educación, México, Fondo de Cultura Económica.

Tedesco, J. C. (2012), Educación y justicia social en América Latina, Buenos Aires, Fondo de Cultura Económica/ Universidad de San Martín.

Universidad Autónoma de Baja California (UABC) (2012), "Proyecto de modificación del programa de licenciatura en ciencias de la educación", <http:/ /fch.mxl.uabc.mx/ wp-content/uploads/2015/04/proyecto-modificacioneducacion.pdf> [Consulta: diciembre de 2015].

\section{Cómo citar este artículo:}

Cordero-Arroyo, Graciela y Ana-María Salmerón-Castro (2017), “El Servicio Profesional Docente y las licenciaturas en ciencias de la educación. Elementos para el análisis", , en Revista Iberoamericana de Educación Superior (RIES), México, UNAM-IISUE/Universia, vol. VIII, núm. 23, pp. 3-24, [consulta: fecha de última consulta]. 